\title{
Case-based analysis in user requirements modelling for knowledge construction
}

Article

Accepted Version

Sun, L. and Mushi, C. (2010) Case-based analysis in user requirements modelling for knowledge construction.

Information and Software Technology, 52 (7). pp. 770-777. ISSN 0950-5849 doi:

https://doi.org/10.1016/j.infsof.2010.03.010 Available at https://centaur.reading.ac.uk/5737/

It is advisable to refer to the publisher's version if you intend to cite from the work. See Guidance on citing.

Published version at: http://www.elsevier.com/locate/infsof

To link to this article DOI: http://dx.doi.org/DOI:10.1016/j.infsof.2010.03.010

Publisher: Elsevier

All outputs in CentAUR are protected by Intellectual Property Rights law, including copyright law. Copyright and IPR is retained by the creators or other copyright holders. Terms and conditions for use of this material are defined in the End User Agreement.

\section{www.reading.ac.uk/centaur}

\section{CentAUR}

Central Archive at the University of Reading 
Reading's research outputs online 


\title{
Case-Based Analysis in User Requirements Modelling for Knowledge Construction
}

\author{
Lily Sun and Cleopa John Mushi \\ School of Systems Engineering \\ University of Reading, RG6 6AY, UK \\ Email addresses: lily.sun@reading.ac.uk, c.j.s.k.mushi@reading.ac.uk
}

\begin{abstract}
Context: Learning can be regarded as knowledge construction in which prior knowledge and experience serve as basis for the learners to expand their knowledge base. Such a process of knowledge construction has to take place continuously in order to enhance the learners' competence in a competitive working environment. As the information consumers, the individual users demand personalised information provision which meets their own specific purposes, goals, and expectations.

Objectives: The current methods in requirements engineering are capable of modelling the common user's behaviour in the domain of knowledge construction. The users' requirements can be represented as a case in the defined structure which can be reasoned to enable the requirements analysis. Such analysis needs to be enhanced so that personalised information provision can be tackled and modelled. However, there is a lack of suitable modelling methods to achieve this end. This paper presents a new ontological method for capturing individual user's requirements and transforming the requirements onto personalised information provision specifications. Hence the right information can be provided to the right user for the right purpose.

Method: An experiment was conducted based on the qualitative method. A medium size of group of users participated to validate the method and its techniques, i.e. articulates, maps, configures, and learning content. The results were used as the feedback for the improvement.

Result: The research work has produced an ontology model with a set of techniques which support the functions for profiling user's requirements, reasoning requirements patterns, generating workflow from norms, and formulating information provision specifications.

Conclusion: The current requirements engineering approaches provide the methodical capability for developing solutions. Our research outcome, i.e. the ontology model with the techniques, can further enhance the RE approaches for modelling the individual user's needs and discovering the user's requirements.
\end{abstract}

Keywords: user requirements profiling, personalised information provision, requirements pattern matching, case-based requirements reasoning, norms

\section{Introduction}

Learning is a process of knowledge construction in which users deploy information from various sources to achieve personal learning goals. In the process the user is engaged with the inquiry by applying their cognitive styles and background knowledge as well as sharing the experience in the learning community $[1,2]$. To support effectively the knowledge construction, one of the critical success factors is to provide the right information in the right format for the right user [3]. Such information provision requires the precise understanding of the user's needs which can be represented in a requirements model. In order to achieve this goal, the requirements engineering (RE) and knowledge management (KM) can aid the requirements analysis and modelling. Various modelling methods, from structured to object-oriented, and from soft to formal, are widely used for requirements analysis [4, 5]. They facilitate the requirements engineering activities for developing domain descriptions. Within a scoped domain of application, the functional behaviour of stakeholders and 
their interaction with the information system can be defined. Furthermore, the information required by the stakeholders can be represented in the data models which support the data manipulation. These techniques are useful for the domain where the problems are well understood. For the problem domains with a greater degree of ambiguity and subjectivity, ontology modelling from KM is considered suitable to enhance the capability of requirements analysis [6, 7]. The ontology model can explicitly capture the domain concepts, social relationships, and temporal aspects as well as cultural constraints in social experiences [8,9]. However, the analysis results produced by these methods are normally represented not in an integrated manner.

To address these issues, this research extends the work done in [7] by developing a new method which enables the complex information provision services to be represented in an ontology model. In this model, a number of techniques perform the analysis of 1) articulating users needs and capturing the user requirements in a profile; 2) mapping the user requirements onto information dimensions in an information space through a reasoning process; 3) configuring the dimensions of the information space in to information provision specifications; and 4) organising information objects in a repository to enable information provision specifications, discovering and retrieving suitable content. The method defines constraints through a norm construct which systemically controls the information provision process.

The remainder of the paper is organised as follows: section 2 discusses the complexity of modelling personalised users' requirements. The characteristics of information provision to support effective knowledge construction is also described in relation to the specific information processing means. The suitability of using case-based reasoning (CBR) is examined for an appropriate reuse of the user requirements in the repository. Section 3 details the method and techniques which facilitate the requirements analysis process. Section 4 validates the method by applying it through a case study and section 5 draws conclusion and recommendations for further work.

\section{Related work}

The information provision for personalised knowledge construction has been addressed by Constructivism [10, 11, 12] and Semiotics [13, 14]. From their perspectives, learning is driven by the user in a situated environment. The requirements elicitation and analysis, therefore, should focus on understanding user's behaviour and identifying the requirements patterns in problem domains of knowledge construction. The requirements articulation has been examined in relation to enhance the user's competence and experience when they are engaged in the learning process [15].

\subsection{Approaches for knowledge construction}

Two instructional design approaches are commonly used to support the knowledge construction, i.e. one-to-many and one-to-one approaches $[16,17,18]$. The former focuses on the expected learning achievement from the learning service provider's perspective. The content in a subject domain is created and delivered to many users. It results often in irrelevant content and too much content being offered to the individual users. This approach is criticised because individual user's preferences and prior knowledge are not taken into account during a design of the information provision. The latter values the alignment between the individual user's learning goal and the organisational strategy and expectation $[1,19]$. This approach is expected to enable the individuals to develop their competence and enhance their performance based on what is required at the time by the workplace [20]. Therefore, it should be capable of recognising and differentiating the learning needs, including cognitive styles, competence and experience, prior-knowledge, and aptitudes.

\subsection{Users cognitive characteristics in knowledge construction}

An examination of users' behaviours in a knowledge construction process reveals that they apply personal learning methods together with various cognitive styles as means for accessing, perceiving, 
and processing new information [21, 22]. Myers and McCaulley [23] and Jung [24] classify several complementary cognitive styles in Myers-Briggs Type Indicator (MBTI). MBTI is a well-tested instrument in both industry and academia for understanding and assessing people's personality and the way they interact with new information. Further to that, Fleming and Mills [25] developed a framework for Visual (V), Auditory (A), Kinaesthetic (K) and Read/Write (R), i.e. VARK, which defines sensory features of learners. Features in cognitive style are defined in [7] and constitute individual's personal information requirements (PIR) as formally described in (1).

$$
P I R=(K C A \times I I \times P I \times P k)
$$

where

$$
\begin{aligned}
& K C A=\{J, P\} \times\{F, T\}=\{(J, F),(J, T),(P, F),(P, T)\}, \\
& I I=\{E, I\} \times\{S, N\}=\{(E, S),(E, N),(I, S),(I, N)\}, \text { and } \\
& P I=\{p(V, A, R, K) / \phi\}
\end{aligned}
$$

Knowledge construction approach (KCA) consists of four types: Feeler (F), Judger (J), perceiver $(\mathrm{P})$, and thinker (T). Interpreting Information (II) describes a way, such as extravert (E), introvert (I), sensing (S), and intuitive $(\mathrm{N})$, by which people understand a subject. Perceiving information (PI) specifies the format preference of information content such as visual, auditory, kinaesthetic or reading/writing. In addition to these features, prior-knowledge $(P k)$ is considered to measure user's knowledge gap such as good, enough, or poor, on a specific subject [26]. PIR features are associated with the description of content in the content repository. They also form the features of pedagogy for what kind of learning content are suitable, in what sequence the content should be organised, and how the organised content ought to be presented. This means that PIR can be used to devise content design strategies $(D e S)$ which are grouped in four patterns as in (2).

$$
\operatorname{DeS} \subset(K C A \times P k)=\{\langle(k, c), p\rangle \mid(k, c) \in K C A, p \in P k\}
$$

where

$$
\begin{aligned}
& D e S_{\text {pull }} \subset(\{(P, T), \text { enough }\} \cup\{(P, T), \text { good }\} \cup\{(P, F), \text { good }\}) \\
& D e S_{\text {push }} \subset(\{(J, F), \text { poor }\} \cup\{(J, T), \text { poor }\} \cup\{(J, F), \text { enough }\}) \\
& D e S_{\text {pull-push }} \subset(\{(P, T), \text { poor }\} \cup\{(P, F), \text { enough }\} \cup\{(J, T), \text { good }\}) \\
& D e S_{\text {push-pull }} \subset(\{(P, F), \text { poor }\} \cup\{(J, T), \text { enough }\} \cup\{(J, F), \text { good }\})
\end{aligned}
$$

$D e S_{p u l l}$ is concerned with narrowcasting information delivery. A typical scenario is that of a piece of information that is provided on demand. This strategy is suitable for users who have enough or good level of prior knowledge to a subject domain. These users do not normally prefer to be overloaded with information that may increase deficiency of the knowledge construction by the "noise" of information.

$D e S_{\text {push }}$ in contrast, considers that information provision serves the common interests to the user with pre-defined information content and navigations. A typical scenario is of a user preferring to be provided with all the relevant information and require constant guidance and notifications of deadlines. The blended strategies $D e S_{\text {pull-push }}$ and $D e S_{\text {push-pull }}$ put the stress on flexibility for users to navigate information content at appropriate level of details according to their level of priorknowledge.

DeS reflects the pedagogical strategies which will be used for a formulation of information provision specifications [27]. Consequently, the specifications will be used to discover information content from the repositories and assemble the content in a package. The searchable information 
content is structured in a form of objects which can be configured and reconfigured according to the individual's needs.

\section{Requirements patterns matching}

User requirements can be represented in specific structures. These structures may follow certain patterns which can be used to discover similarity of the existing requirements to a new request [28]. Such analysis can be carried out by case-based reasoning (CBR) [29] whereby the user requirements can be represented as a case $C$ comprising a problem space of $p l$ and a solution space of $s$ defined as $C \subset(p l, s)$ and represented in (3).

$$
p l \subset \sum_{i=1}^{n} f_{i}\left(v_{i}, w_{i}\right)
$$

where

$f_{i}\left(v_{i}\right)$ : a description (value) of the problem feature

$f_{i}\left(w_{i}\right)$ : a weight (usually between 0 and 1 ) of the problem feature

Each $f_{i}\left(v_{i}, w_{i}\right)$ of $p l$ corresponds to a feature of cognitive style, e.g. KCA, II, $P I$, and $P k$, and a weight according to its degree of significance to the knowledge construction. Figure 1 shows a structure of the matching process with $C^{I}$ as an input case and $C^{R}$ as a case in the repository which is a database that holds all cases. In the matching process, if $p l$ of $C^{I}$ matches $p l_{i}$ of $C^{R}$, then, solution space $s$ of $C^{I}$ can be derived by reusing $s_{i}$ of $C^{R}$. In this work a target problem of $C^{I}$ is an inputted new case which will be reasoned against the existing cases in the case repository [30, 31]. To derive the solution space, $C^{I}$ is compared against $C^{R}$ through their corresponding features $f_{i}$ and $f_{i}^{R}$ by executing the Nearest Neighbour (NN) function of $\operatorname{sim}\left(f_{i}, f_{i}{ }^{R}\right)$ according to the rules specified in the knowledge base. The resultant similar case (retrieved case), satisfies a threshold $\delta$ of the similarity measure defined in (4).

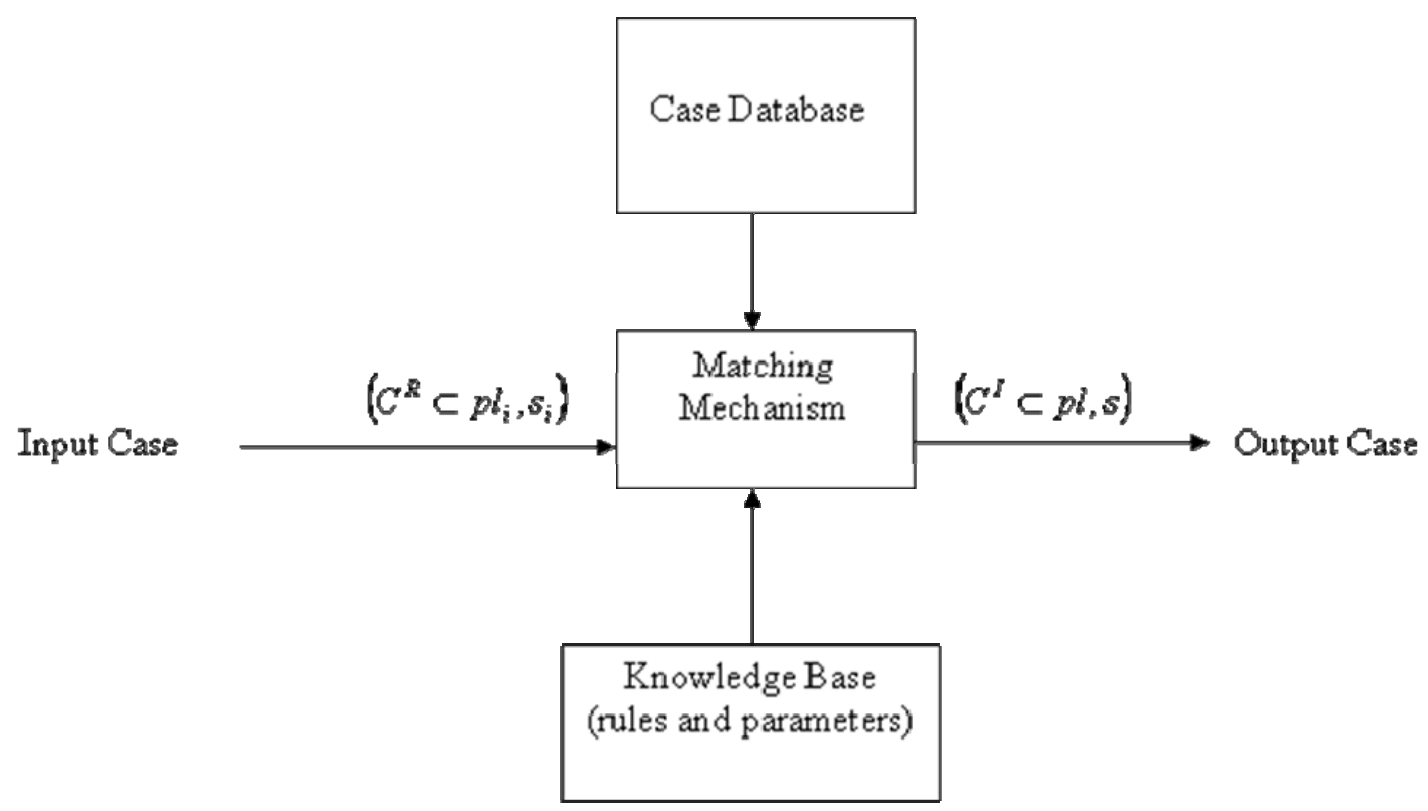

Fig. 1. Case matching process. The input case $C^{I}$ is matched against a case $C^{R}$ in the repository using rules specified in the knowledge base to derive the solution space, $s$. 


$$
\operatorname{sim}\left(f_{i}, f_{i}{ }^{R}\right)=\left\{\begin{array}{l}
1, \text { given } f_{i}=f_{i}{ }^{R} \\
0, \text { given } f_{i} \neq f_{i}{ }^{R}
\end{array}\right.
$$

where

$f_{i}$ and $f_{i}{ }^{R}$ : attributes for a new case and retrieved case respectively.

$\operatorname{sim}\left(f_{i}, f_{i}^{R}\right)$ : A similarity measure (as \%) between the two cases for the feature $f_{i}$.

With CBR, a temporal aspect of the case is another critical factor in solving a problem. Amongst the alternative solutions, the temporality is often crucial to the problem space in the industrial nature whose processes might yield different solutions at different times [32]. However, our work considers that weighting is important as it indicates the degree of importance to KCA, II and PI. The value of $K C A$ influences greatly on the adoption of the value of $I I, P I$ and $P k$. Each $P I R$ can be considered as a case and the elements of PIR correspond to the problem features in cases. The cases can further be used to form patterns of information provision specifications.

\section{Knowledge Construction Model}

An ontology model in Figure 2, based on the principles of [13, 27] is designed to represent the personalised information provision in the application domain of lifelong learning. In this model, the affordance of articulates and maps transform the user needs onto the information provision requirements in the form of $I S_{\text {selection, }} I S_{\text {sequencing }}$ and $I S_{\text {presentation. The affordance of configures will then }}$ produce the information provision specifications based upon which the personalised content can be packaged.

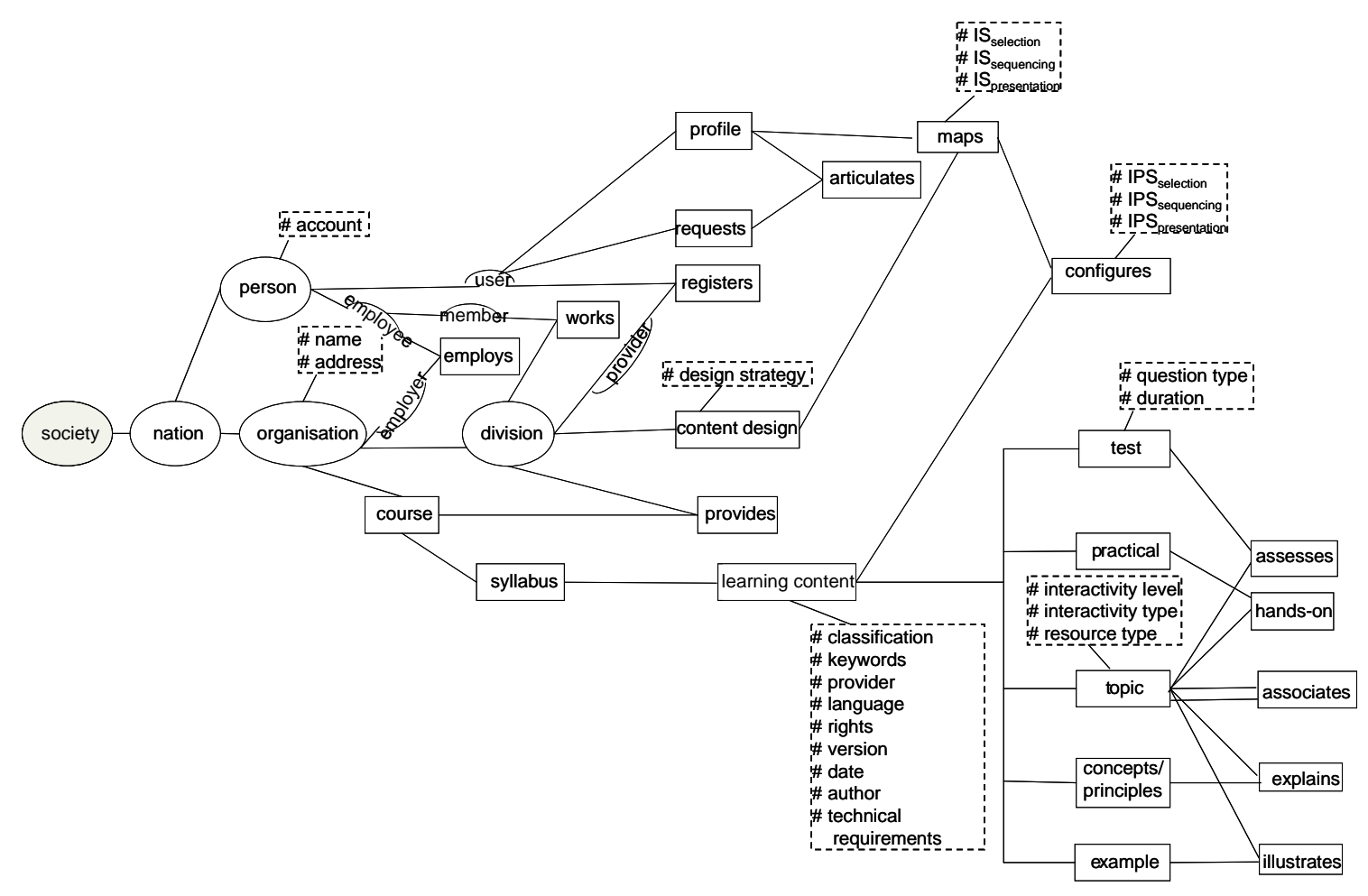

Fig. 2. The ontology model for knowledge construction. User requirements are articulated and captured in the profile, mapped onto information dimensions in an information space using the reasoning process, configured into information provision specifications and then organised in a repository to enable information provision specifications, discovering and retrieving suitable content. 


\subsection{Articulation of user's needs}

A person as user in the ontology chart can express requests for information from the content repository. This request will be processed by the affordance of articulates which also requires the antecedent of profile to provide the user preference, competency, and portfolio of qualifications and experiences [33, 34, 35]. The profile in Figure 3 formally defines the user requirements. Personal_Information captures individual's information which is obtained from the process of registers. The Security defines authentication for users. Learning_ Monitor consists of information for tracking 1) a learning plan (e.g. activities and time frame) for the chosen subject in supporting the knowledge construction process; and 2) feedback generated throughout this process. The monitoring information will be used to revise the solution of $C^{I}\left(s^{I-1}\right)$ into $C^{I}\left(s^{I}\right)$. The user progress and achievements are stored in the Performance which will further assist a formulation of competencies. Interests stores users interests which can assist instructional provision in terms of useful planning for short-term and long-term self-development, and collaborative learning based on the interests.

Portfolio contains a collection of references about users, such as learning history, qualifications, strengths, achievements, and experiences. Portfolio assists the articulation of the user prior knowledge. Information_Requirements includes two categories, Preferences which corresponds to PIR and Content_Requirements $(C R)$ which consists of the parameters: (personal_goal, subject, difficulty_level, resource_type, language) which represent the user's learning request. For example, one desires to study a subject of information systems design methods at the advanced level. The content should be in English. The goal for acquiring such knowledge aims to become a competent business analyst. This request should be satisfied by providing the right information content in the subject domain. $C R$ is normally coupled with $P I R$ for personalising the requested information content. Both $P I R$ and $C R$ form the problem features $\left(f_{1} \ldots f_{n}\right)$ of the case:

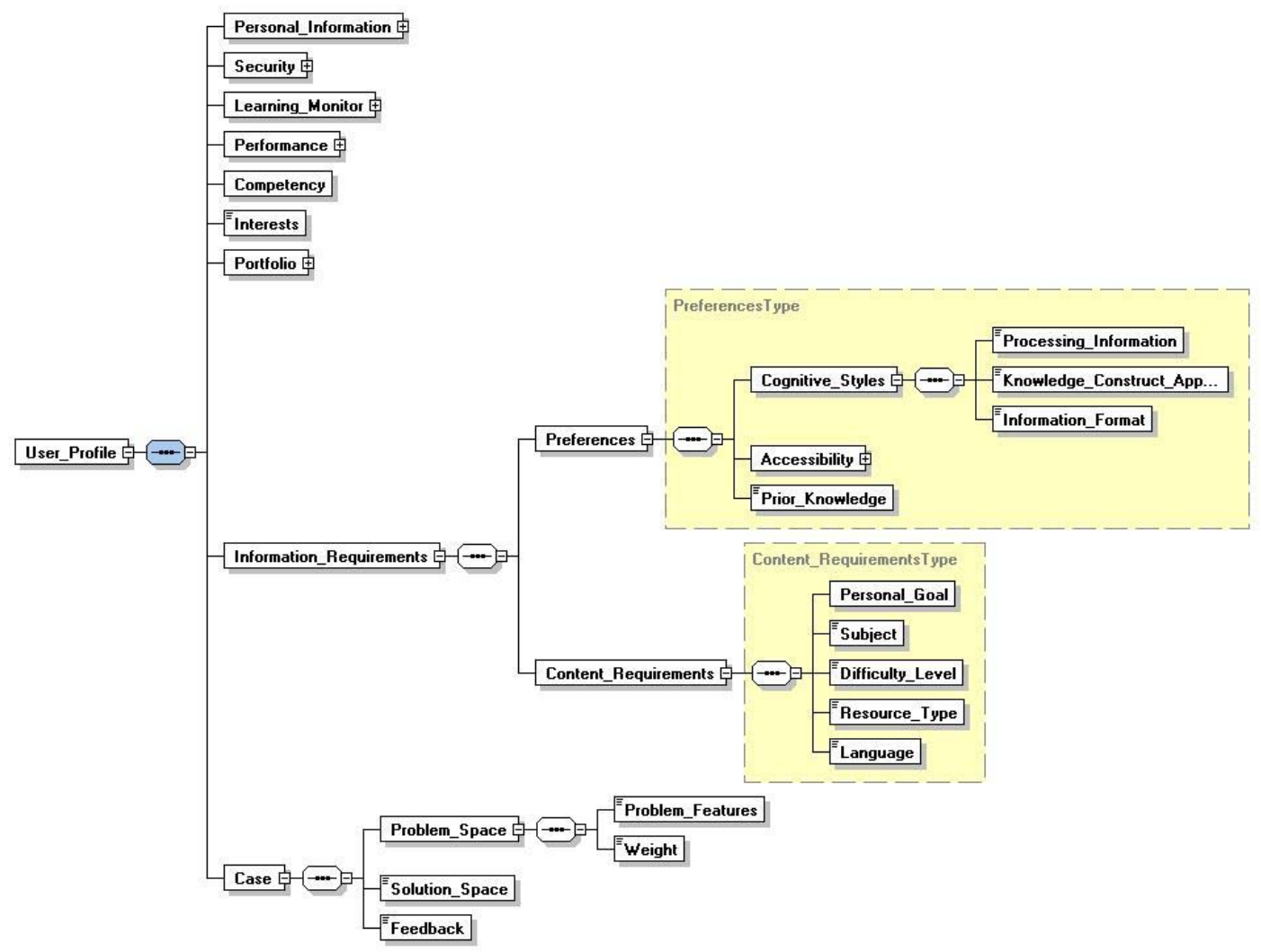

Fig. 3. User profile schema. This models user's information requirements 


$$
\begin{aligned}
C= & ((\text { KCA,0.4), }(I I, 0.3),(P I, 0.3),(P k, 0.3),(\text { personal_goal,0.1), (subject,0.1), (difficult_level,0.3), } \\
& (\text { resource_type,0.1),(language,0.4), s })
\end{aligned}
$$

In this description, PIR features as well as CR.difficult_level and CR.language are considered as important conditions for a case to be reasoned for matching with user requirements patterns. The parameters in Case will be used as the future reference to derive information space specifications in the maps process. The articulate process accomplishes certain activities to elicit user requirements and to formulate PIR [28].

\subsection{Formulation of information provision requirements}

In the ontology chart, the antecedence of Profile and Content Design can afford the concept of maps. The maps carries out a process to transform the parameters in PIR and $C R$ on to information provision requirements. The information provision requirements characterise 1 ) what content should be selected; 2) how the selected content should be navigated based on pedagogical instructions; and 3) how the content and the navigations should be presented to the user in accordance with their personalised requirements. These three aspects of the information provision requirements are expressed formally in (5), (6) and (7).

$$
\begin{aligned}
& I S_{\text {selection }} \subset C R \cup(I I \times P I \times P k) \\
& I S_{\text {sequencing }} \subset C R \cup D e S \\
& I S_{\text {presentation }} \subset P I
\end{aligned}
$$

Subsequently, these three dimensions constitute a solution $s$ for a case, i.e. $s \subset\left(I S_{\text {selection }}, I S_{\text {sequencing }}, I S_{\text {presentation }}\right)$

The process of maps is governed by a set of executable norms which describe the maps workflow. A StartNorm and FinishNorm in Figure 4 define the maps process existence and its cease. Within a life span of the process, two operational norms in Figure 5 are also defined to control the workflow of maps.

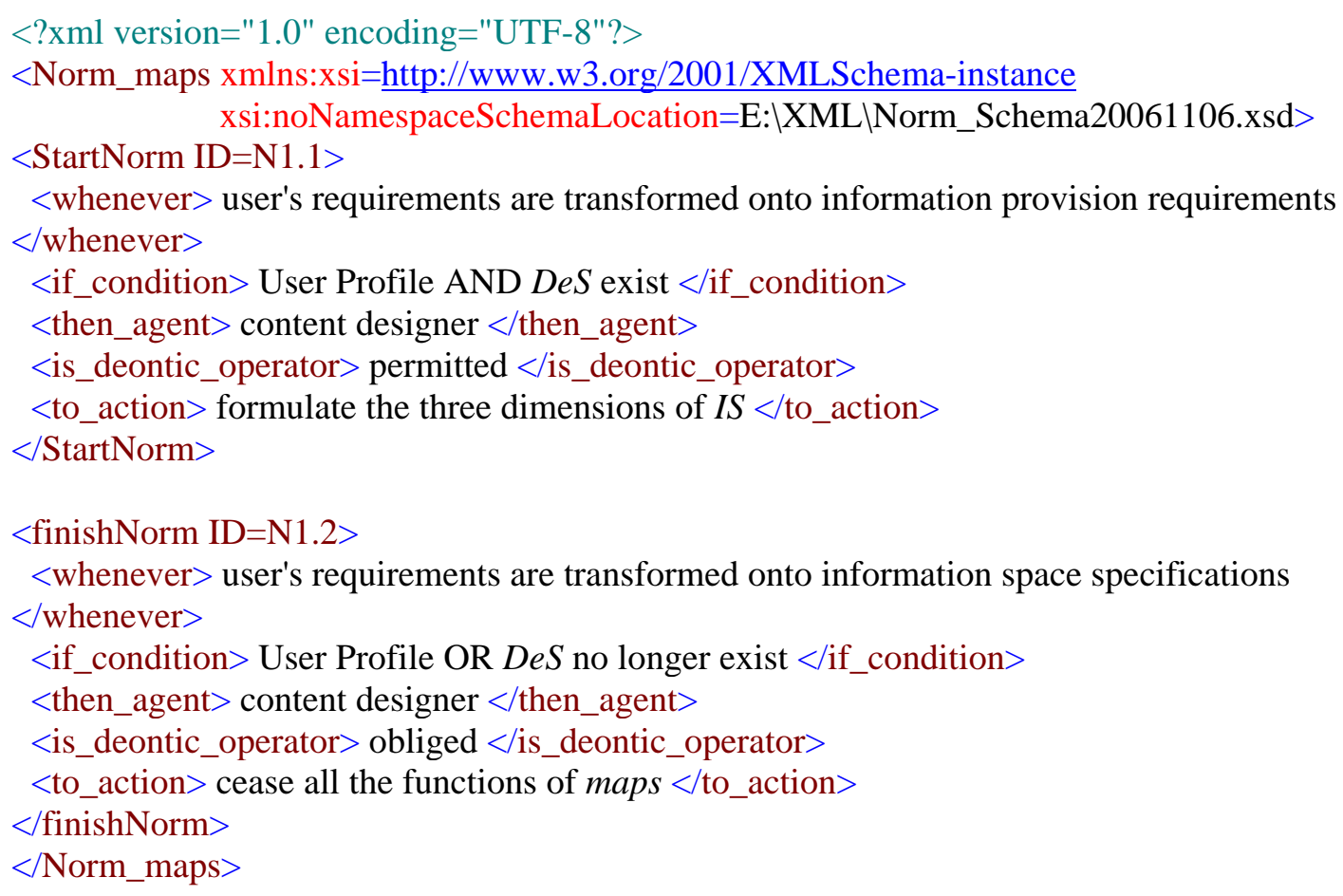

Fig. 4. The norms defining the existence of maps. 
N1.3 and N1.4 are the operational norms which specify two algorithms for creating a solution of $s$ for the new case. The algorithm in N1.3 generates the three dimensions of IS and the suitable pedagogical strategy of DeS when there are no matching user requirements in Profile to solve $C^{I}\left(f_{i}{ }^{I}, s\right)$. N1.4 creates $s$ for the new case by reusing and revising the solution of the most similar existing case. The computed new case will be stored in Profile for the future use.

\section{Validation and Discussion}

The research presented in this paper is a part of the university's initiative of e-learning. The method and its techniques described have been applied in the transformation from traditional learning paradigm to constructivist approaches in e-learning. The experiment of the techniques in the ontology chart, such as articulates, maps, configures, and learning content, was carried out in a group of 61 users. Information Systems Engineering was chosen as the subject domain in which the user is supported for knowledge construction. The content of the subject is designed in the form of learning objects which are stored in the content repository. The learning objects are capable of facilitating the

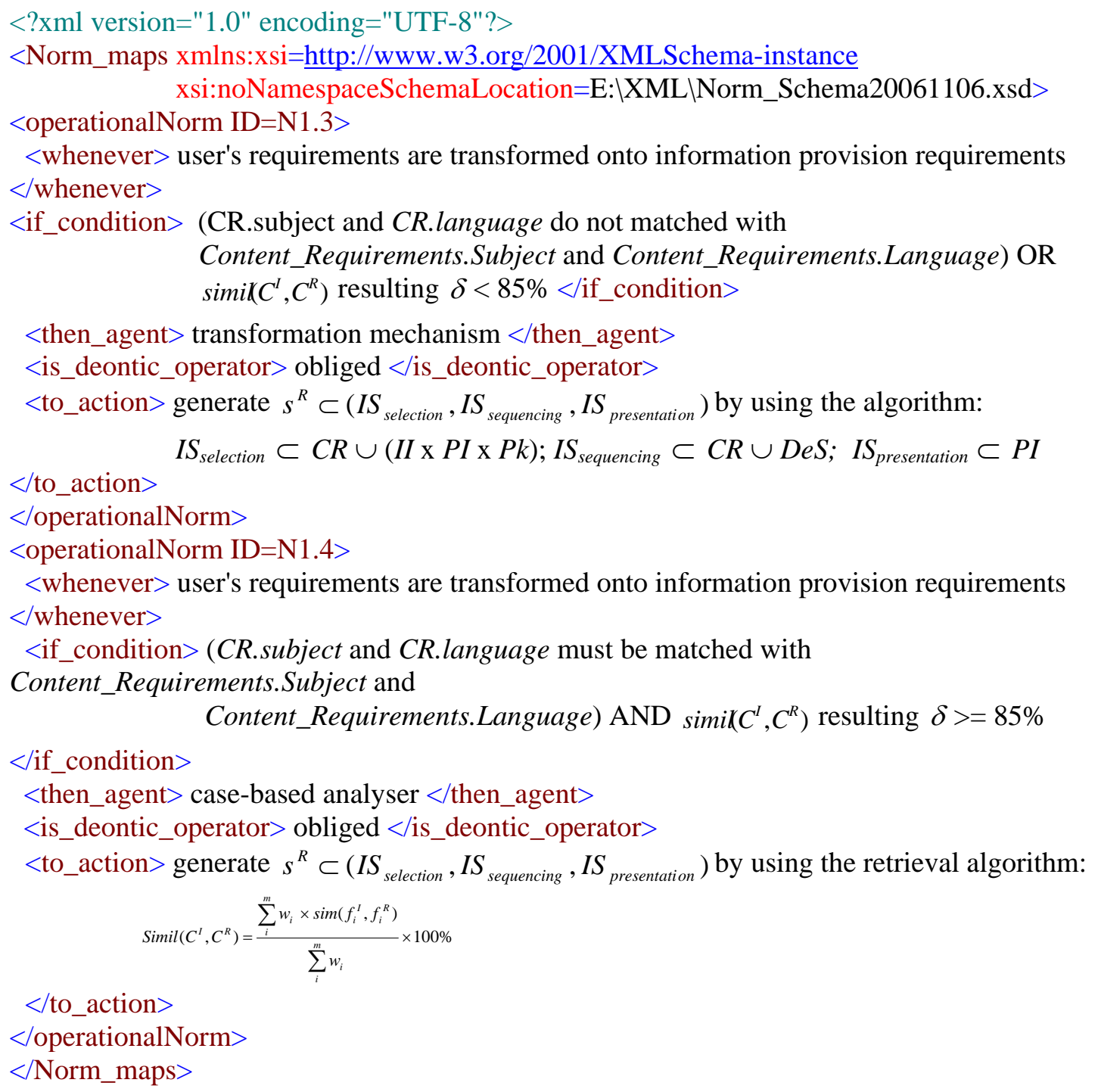

Fig. 5. The norms defining the functions of maps. 
content selection, sequencing and presentation for a personalised learning package.

A case base is created in a structure of \{problem features, solution, feedback\} (see Figure 6). The problem features contain the parameters of PIR and $C R$ as well as their associated weightings. In articulates, two types of assessment, i.e. Cognitive Style Assessment (CSA) and Prior Knowledge Assessment (PKA) [9] are used for capturing the PIR for the user. This user is also encouraged to express a request in $C R$ for gaining the knowledge in the subject domain to pursue the learning goal. For example, the user, $C^{62}$, requested to learn UML to become a business analyst. Based on this request, the user's profile is examined in conjunction with the assessment of his cognitive style and prior knowledge. His $P I R$ and $C R$ were resulted in:

$$
\begin{aligned}
& P I R=((P, T),(E, S),(V, K), \text { enough }) \\
& C R=(\text { bu sin ess analyst }, U M L, \text { advanced }, \text { topic }, \text { English })
\end{aligned}
$$

The solution, i.e. $s$, indicates that this user requires minimal constraints on the navigation between different parts of content, because he is an active thinker and prefers to acquire the UML topic in a less constrained manner. Although he has already some basic knowledge about the topic, he still wishes to attend the topic at the advanced level with some pedagogical guidance. The $P I R$ and $C R$ were further used to create his case of $C^{62}$ as shown in Figure 6. Based on how they significantly impact on learning, all the 9 problem features are assigned with weighting as very high priority (0.4), high priority (0.3) or low priority (0.1).

The maps executes the case matching process by taking $C^{62}$ as input and matching it against all the cases in the case-base. By adopting [36], 10 cases are retrieved from the case-base and presented in Table 1. N1.3, which specifies a threshold at $\delta>=85 \%$, decides that $C^{30}$ and $C^{15}$ are the most relevant cases and their $S$ can be adopted by $C^{62}$. In order to evaluate this decision, Normalized Discounted Cumulative Gain (NDCG) is used [37]. The overall matching against each relevant case in Table 1 is processed by Discounted Cumulative Gain (DCG) to identify their gain or usefulness in their respective positions, $p$, in the rank. However, the DCG $_{p}$ does not explicitly distinguish which $f_{i}$ in the compared cases are not matched, although the overall matching may result in the same percentage. To overcome such ambiguity, the user was given the opportunity to judge the retrieved cases for suitability. Each retrieved case is scaled as highly relevant (3), fairly relevant (2), marginally relevant (1) or irrelevant (0) [38] from the user viewpoint toward the learning.

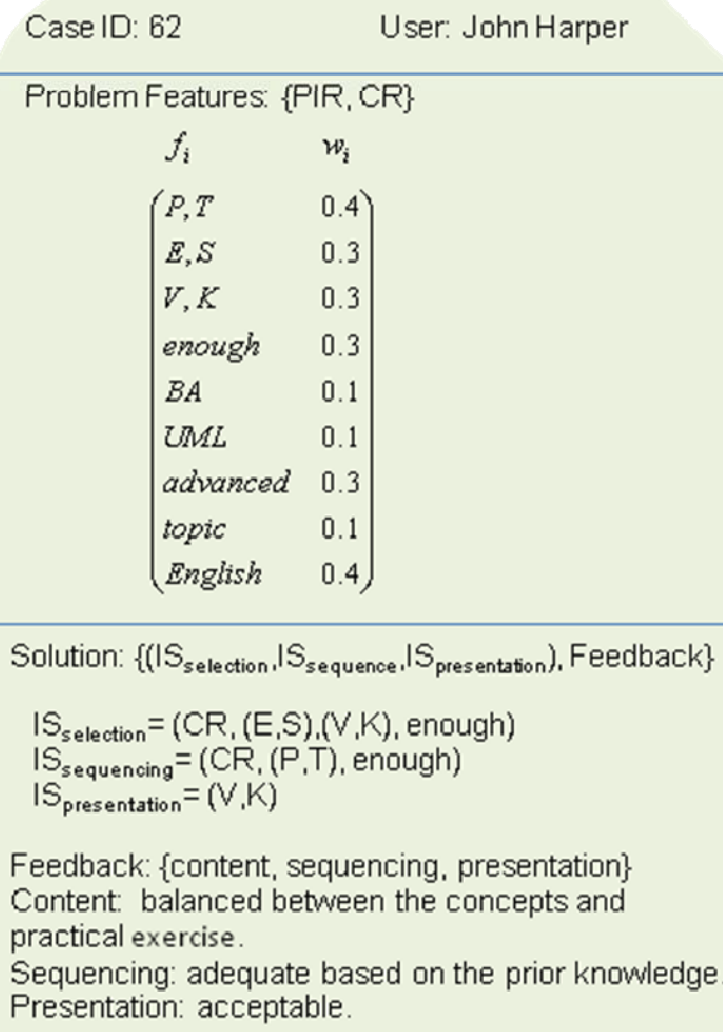

Feedback: \{content, sequencing, presentation\} Content: balanced between the concepts and practical exercise.

Sequencing: adequate based on the prior knowledge Presentation: acceptable

Fig. 6. A representation of the case 
Table 1

The ranking list of the relevant cases

\begin{tabular}{ccccccc}
\hline Relevant Case & \multicolumn{1}{c}{$D C G_{p}$} & \multicolumn{3}{c}{ IDCG } \\
& $\begin{array}{c}\text { Overall Match } \\
(\%)\end{array}$ & Rank $_{p}$ & Log $_{2}$ Rank & Value & User Scale & Ideal Rank $_{p}$ \\
\hline Simil $\left(C^{62}, C^{30}\right)$ & 91.30 & 1 & 0.0000 & 3.0000 & 3 & 1 \\
$\operatorname{Simil}\left(C^{62}, C^{15}\right)$ & 91.30 & 2 & 1.0000 & 3.0000 & 3 & 2 \\
$\operatorname{Simil}\left(C^{62}, C^{52}\right)$ & 82.61 & 3 & 1.5850 & 0.6309 & 1 & 4 \\
$\operatorname{Simil}\left(C^{62}, C^{6}\right)$ & 73.91 & 4 & 2.0000 & 0.5000 & 1 & 5 \\
$\operatorname{Simil}\left(C^{62}, C^{46}\right)$ & 69.57 & 5 & 2.3219 & 0.0000 & 0 & 7 \\
$\operatorname{Simil}\left(C^{62}, C^{28}\right)$ & 69.57 & 6 & 2.5850 & 0.7737 & 2 & 3 \\
$\operatorname{Simil}\left(C^{62}, C^{8}\right)$ & 65.22 & 7 & 2.8074 & 0.0000 & 0 & 8 \\
$\operatorname{Simil}\left(C^{62}, C^{10}\right)$ & 65.22 & 8 & 3.0000 & 0.0000 & 0 & 9 \\
$\operatorname{Simil}\left(C^{62}, C^{23}\right)$ & 65.22 & 9 & 3.1699 & 0.3155 & 1 & 10 \\
$\operatorname{Simil}\left(C^{62}, C^{24}\right)$ & 65.22 & 10 & 3.3219 & 0.0000 & 0 & 6 \\
& & & Total DCG & 8.2201 & & \\
\hline
\end{tabular}

The user scales are then used by Ideal Discounted Cumulative Gain (IDCG) to subjectively create the ideal rank which differs from $D_{C G}$. The result of $N D C G=0.9581$ indicates that the reasoning process has effectively retrieved the relevant cases for discovering the user requirements formulated in $C^{62}$. The experiment has further processed three more new cases. Each NDCG result validated the retrieval performance (see Table 2). All the results have consistently established that $\partial \geq 85 \%$ is appropriate threshold value to determine highly relevant retrieved cases.

In a knowledge construction process, the problem features may evolve over time. Therefore, the existing training cases may be out-of-date for reasoning. The method needs to incorporate a selfevolving capability. The weighting to each problem feature cannot be adjusted according to the subject classification in the current method. For example, the user may apply the cognitive style differently from subject to subject (e.g. engineering or social science). The cognitive style is sometimes determined by the prior knowledge when the new knowledge is being constructed. To improve this end, the current weighting system will incorporate a control mechanism which enables a critical view of the priority settings for case matching levels and also the effect that can be imposed on the threshold value.

A feedback mechanism can be integrated in the ontology model. This mechanism can perform a tracking function to monitor the user's behaviour through the knowledge construction pathway. If a user wishes to change the way by which the information content can be provided during the knowledge construction process, those changes will appropriately be incorporated in the information provision specifications. Requirements pattern mining algorithms are also necessary for improving the efficiency and accuracy of requirements matching. All the techniques in the method will be designed in a set of CASE tools which can systematically aid the creation and documentation of an ontology model.

The functions and workflows in the model can be formally and logically described. The outcome produced by the CASE tools can then be used as input to the learning management systems for

Table 2

Evaluating effectiveness of case-based retrieval system

\begin{tabular}{cccccc}
\hline Evaluation method & $C^{62}$ & $C^{63}$ & $C^{64}$ & $C^{65}$ & Average \\
\hline NDCG & 0.9581 & 0.9739 & 0.8503 & 0.9662 & 0.9371 \\
\hline
\end{tabular}


personalised information discovery and provision.

\section{Conclusion}

Personalised information provision can be formally represented to support the knowledge construction. In this paper we have suggested the use of ontology model to conceptualise the semantic units which define the process from capturing the user's needs to employing the personalised information provision specifications for a delivery of information content. The norms embedded in the semantic units execute this process that ensures the right information for the right need. The three key processes in the method, i.e. articulates, maps and configures, perform various necessary functions: 1) to facilitate the user profiling and articulate PIR and CR to form cases; 2) to execute the norms for analysing the user requirements and information provision specifications; 3) to inference cognitive features for personalisation; 4) to generate requirements patterns; and 5) to formulate requirements specifications. The application of the method is validated by adopting the NDCG technique. The result reflects the adequacy of modelling the information provision requirements and the suitability of CBR to support the user requirements analysis. The method is being undertaken for critical improvement which will enable its techniques to be used in other application domains.

\section{References}

[1] J.S. Brown and A.R. Adler, Minds on Fire: Open Education, the Long Tail, and Learning 2.0, EDUCAUSE Review, 43(1), 2008, pp. 16-32.

[2] K. Wright, Personal Knowledge Management: Supporting Individual Knowledge Worker Performance, Knowledge Management Research and Practice, 3, 2005, pp. 156-165.

[3] P. Brusilovsky, Adaptive hypermedia, User Modelling and User Adapted Interaction. In Kobsa A. (ed.), Ten Year Anniversary Issue, 11(1/2), 2001, pp. 87-110.

[4] P. Laplante, Requirements Engineering for Software and Systems, Redmond, WA: CRC Press, 2009.

[5] B. Nuseibeh and S. Easterbrook, Requirements Engineering: a Roadmap, in Proceedings of the Conference on the Future of Software Engineering, Limerick, Ireland, 2000, pp. 35-46.

[6] A. Gómez-Pérez, M. Fernandez-Lopez and O. Corcho, Ontological Engineering-with examples from the areas of Knowledge Management, e-Commerce and the Semantic Web, SpringerVerlag, London, 2004.

[7] L. Sun and K. Ousmanou, Articulation of Information Requirements for Personalised Knowledge Construction, Journal of Requirements Engineering, 11(4), 2006, pp. 279-293.

[8] A. Lau and E. Tsui, Knowledge Management Perspective on e-Learning Effectiveness, Knowledge-Based Systems, 22(4), 2009, pp. 324-325.

[9] K. Liu, L. Sun and J. Fu, Ontological Modelling for Content Management and Provision, Journal of Information \& Software Technology, 50(11), 2008, pp. 1155-1164.

[10] P.C. Honebein, T. Duffy and B. Ishman, Constructivism and the Design of Learning Environment: Context and Authentic Activities for Learning. Design Environments for Constructivist Learning, New York, Springer-Verlag, 1993, pp. 87-108.

[11]D.H. Jonassen, Objectivism versus constructivism: Do we need another philosophical paradigm? Educational Technology Research and Development, 39(1), 1991, pp. 5-14.

[12] A. Nanjappa and M. M. Grant, Constructing on Constructivism: The Role of Technology, Electronic Journal of the Integration of Technology in Education, 2(1), 2003, pp. 38-55.

[13] K. Liu, Semiotics in Information Systems Engineering, Cambridge, Cambridge University Press, 2000.

[14] C.S. Peirce, Collected Papers of C.S. Peirce, In C. Hartshorne et al., (eds), Cambridge, Massachusetts, 1935. 
[15] K.-D. Althoff and D. Pfahl, Making Software Engineering Competence Development Sustained through Systematic Experience Management, Managing Software Engineering Knowledge, Springer Verlag, 2003.

[16] C. Estes, Promoting Student-Centred Learning in Experiential Education, The Journal of Experiential Education, 27(2), 2004, pp. 141-161.

[17] C. Reigeluth, Instructional Design Theories and Models: A New Paradigm of Instructional Theory, Lawrence Erlbaum Associates Mahwah, NJ, 1999.

[18] R. Schank, Dynamic Memory: A Theory of Learning in Computers and People, New York: Cambridge University Press, 1982.

[19] Z. Liu, W. Ng and E. Lim, Personalized Web Views for Multilingual Web Sources, IEEE Internet Computing, 2004, pp. 16-22.

[20] J. Cao, J. Wang, K. Law, S. Zhang and M. Li, An Interactive Service Customization Model, Journal of Information and Software Technology, 48(4), 2006, pp. 280-296.

[21] S. Pedersen and D. Williams, A Comparison of Assessment Practices and Their Effects on Learning and Motivation in a Student-Centered Learning Environment. Journal of Educational Multimedia and Hypermedia, 13 (2004) (3), pp. 283-307.

[22] D. Riecken, Personalized Views of Personalisation. Communications of the ACM, 43(8), 2000, pp. 26-28.

[23] I.B. Myers and M.H. McCaulley, Manual: A Guide to the Development and Use of the MyersBriggs Type Indicator, Palo Alto, Consulting Psychologists Press, 1985.

[24] C.G. Jung, Psychological Types or the Psychology of Individuation, Brace, Harcourt, New York, 1923.

[25] N.D. Fleming and C. Mills, Not Another Inventory, Rather a Catalyst for Reflection, To Improve the Academy, 11, 1992, pp. 137.

[26] T. Iyoshi, M. Hannafin and F. Wang, Cognitive Tools and Student-centred Learning: Rethinking Tools, Functions and Applications, Educational Media International, 42(4), 2005, pp. 281-296.

[27] L. Sun, K. Ousmanou and M. Cross, An Ontological Modelling of User Requirements for Personalised Information Provision, Journal of Information Systems Frontiers, 2009.

[28] K. Liu and L. Sun, A Method for Interactive Articulation of Information Requirements for Strategic Decision Support, Journal of Information and Software Technology, 43(2), 2001, pp. 247-263.

[29] A. Aamodt and E. Plaza, Case-based reasoning: Foundational issues, methodological variations and system approaches. Artificial Intelligence Communications, 7(1), 1994, pp. 39-59.

[30] J. Meléndez, J. Colomer and J. Macaya, Case-Based Reasoning Methodology for Supervision, In Proceedings of the European Control Conference, Oporto, Portugal, 2001, pp. 1600-1605.

[31] W. Vollrath and W. Wilke, R. Bergmann, Case-Based Reasoning Support for Online Catalogue Sales, IEEE Internet Computing, 1998, pp. 47-54.

[32] P. Alvis, L. Amaral and J. Pires, Case-Based Reasoning to Adaptive Web-Based Education System, In: Proceedings of the $8^{\text {th }}$ IEEE International Conference on Advanced Learning Technologies, 2008, pp. 260-261.

[33] IEEE Papi, IEEE Public and Private Information, at: www.edutool.com/papi/, 2003.

[34] IMS lip, Learner Information Package Specification, at: imsglobal.org/profiles/index.cfm, 2003.

[35] S. E. Middleton, N. R. Shadbolt and D.C. De Roure, Ontological User Profiling in Recommender Systems. ACM Transaction, Information Systems, 22(1), 2004, pp. 54-88.

[36] C.D. Manning, P. Raghavan and H. Schütze, Introduction to Information Retrieval, Cambridge University Press, 2008, ISBN 978-0521865715.

[37] J. Kalervo, J. Kekalainen, Cumulated gain-based evaluation of IR techniques, ACM Transactions on Information Systems, 2002, 20(4), pp. 422-226. 
[38] P. Vakkari, Elevance and contributory information types of searched documents in task performance, in: N.J. Belkin, P. Ingwersen, M.K. Leong (Eds), Proceedings of the 23rd Annual International ACM SIGIR Conference on Research and Development in Information Retrieval, ACM Press, New York, NY, 2000, pp.2-9. 